\title{
Treatment of Severe Pain and Opioid-induced Constipation: An Observational Study of Quality of Life, Resource Use, and Costs in Sweden
}

Frida Hjalte (D) Gunnel Ragnarson Tennvall · Karl-Olof Welin •

Dagmar Westerling

Received: September 19, 2016 / Published online: November 9, 2016

(c) The Author(s) 2016. This article is published with open access at Springerlink.com

\section{ABSTRACT}

Introduction: Opioid-induced constipation (OIC) is a common and costly side effect of opioid treatment affecting patients' quality of life (QoL). The combination oxycodone/naloxone reduces OIC while providing effective analgesia in patients with moderate to severe pain. The objective of this observational study was to compare health-related quality of life (HRQoL), healthcare resource use, and costs in patients with severe pain who were initially treated with oxycodone and laxatives and then subsequently

Enhanced content To view enhanced content for this article go to.http://www.medengine.com/Redeem/ 2417F0604D2982B1.

F. Hjalte $(\bowtie) \cdot$ G. Ragnarson Tennvall .

Karl-OlofWelin

IHE, The Swedish Institute for Health Economics,

Lund, Sweden

e-mail: fh@ihe.se

D. Westerling

Pain Unit, Department of Anesthesiology, Central

Hospital of Kristianstad, Kristianstad, Sweden

D. Westerling

Section of Anaesthesiology and Intensive Care, Department of Clinical Sciences, Lund University, Lund, Sweden switched to treatment with oxycodone/naloxone.

Methods: Data were collected by means of questionnaires completed by patients with OIC before and after the initiation of oxycodone/naloxone treatment at different clinical centers in Sweden. The questionnaires included questions on healthcare resource use and absence from work and also consisted of the Patient Assessment of Constipation-QoL (PAC-QoL) instrument, the EuroQol five dimensions questionnaire (EQ-5D), the Patient Assessment of Constipation Symptoms (PAC-SYM) instrument, and the Bowel Function Index (BFI).

Results: The analysis included 37 patients. Resource utilization was lower after treatment with oxycodone/naloxone, in terms of both the number of healthcare contacts and the purchases of medicine for the treatment of constipation. According to the BFI score, patients had fewer problems with OIC after the initiation of oxycodone/naloxone. The PAC-QoL score showed a positive change for patients in both the 96-point dissatisfaction index and the 16-point satisfaction index. PAC-SYM scores was lower after the initiation 
of oxycodone/naloxone treatment, indicating fewer constipation-related problems.

Conclusion: Treatment with oxycodone/naloxone had an overall positive effect on patients, consisting mainly of decreasing the severity of the constipation problems, increasing HRQoL, and decreasing the use of healthcare resources.

Funding: Mundipharma AB, Gothenburg, Sweden.

Keywords: Costs; Laxatives; Naloxone; Opioid-induced constipation; Oxycodone; Quality of life; Resource use; Severe pain; Sweden

\section{INTRODUCTION}

Opioids are used for moderate to severe pain caused by cancer [1], trauma, and operations. Opioid therapy may be considered in combination with non-pharmacological and non-opioid therapies for selected patients suffering from severe nonmalignant persistent pain [2]. However, treatment with potent opioids is connected with frequent and problematic side effects [3]. One of the most common side effects is opioid-induced constipation (OIC). In a publication based on patient data from a Swedish noninterventional study, the conclusion was that OIC is a costly complication that also affects the quality of life (QoL) of patients. The complication may also limit effective pain therapy [4].

Oxycodone/naloxone is an opioid analgesic combined with an opioid antagonist in a fixed prolonged-release combination which has been shown to significantly reduce opioid-induced constipation while providing effective analgesia in patients with moderate to severe non-malignant pain [5]. Oxycodone/naloxone has also been confirmed to be well tolerated and efficacious in patients suffering from moderate to severe cancer pain [6].

The objective of the observational study was to compare health-related QoL (HRQoL), healthcare resource use, and costs in patients with severe pain, treated with oxycodone and laxatives, who were subsequently switched to treatment with oxycodone/naloxone.

\section{METHODS}

The study (OXN9513 GAIN) was an observational study of patients with severe pain treated with opioids in Sweden. Patients treated with oxycodone in a stable, adequate dose and time period with symptoms of OIC despite laxatives were enrolled in the study. The participating patients served as their own controls, and data for comparison were therefore collected retrospectively before the initiation of oxycodone/naloxone treatment and after initiation of treatment with oxycodone/naloxone. The prescription of oxycodone/naloxone was clearly separated from the decision to include the patient in the study.

The observational study consisted of two visits, i.e., before (visit 1) and after (visit 2) the initiation of oxycodone/naloxone treatment, covering a total period of 8 weeks, as illustrated in Fig. 1 . The results from visit 1 are thus related to the situation when patients were treated with oxycodone and laxatives, while the results from visit 2 correspond to the situation when patients had switched to treatment with oxycodone/naloxone. In both cases the survey answers deal with the last 4 weeks before the visit, i.e., all collected data were retrospective.

The primary data sources were patient questionnaires, which were filled out by patients with OIC before and after the 


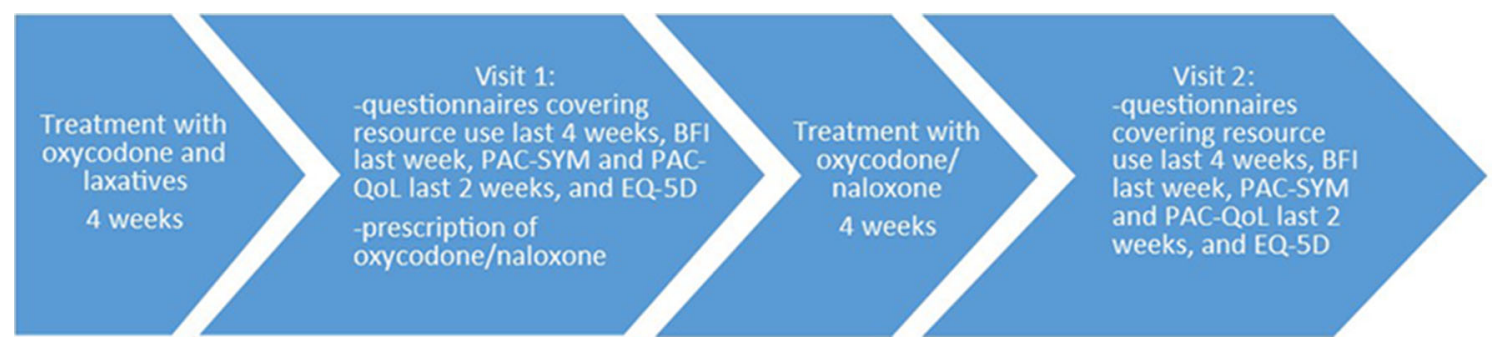

Fig. 1 Observation period

initiation of oxycodone/naloxone treatment, and a case report form (CRF) filled out by the clinical investigator. The CRF contained questions about patient characteristics, cause of pain, drug treatment, adverse events, and classification of pain. The physician also completed the clinician-administered, patient-reported Bowel Function Index (BFI) before and after the initiation of oxycodone/naloxone treatment. The answers to the BFI correspond to the patients' experience of bowel function during the previous 7 days.

Before and after the initiation of oxycodone/naloxone, the patient completed questionnaires with two self-administered disease-specific instruments, namely, the Patient Assessment of Constipation-QoL (PAC-QoL) and the Patient Assessment of Constipation Symptoms (PAC-SYM) instruments. PAC-QoL assesses the degree to which constipation has reduced the patient's QoL during the previous 2 weeks, and the associated PAC-SYM measures the constipation-related symptoms during the same 2 weeks. Patients were also asked to complete the generic quality-of-life instrument EuroQol five dimensions (EQ-5D) together with the EuroQoL visual analog scale (EQ-VAS).

Patients also completed a separate survey at visit 1 and visit 2 that included questions on their resource utilization in the 4 weeks before and after initiation of oxycodone/naloxone treatment, respectively. These questions were aimed at obtaining patient-specific information on constipation and/or pain-related additional visits or telephone contacts with physicians and other caregivers, inpatient care, and purchases of laxatives. The patient survey also included questions on employment, absence from work and ability to work.

The general structure of the reported values from the different instruments and forms consisted of the values before and after the initiation of oxycodone/naloxone and the difference between them. The calculation of the change in HRQoL was performed by subtracting the reported value before the initiation of oxycodone/naloxone from the reported value after the initiation of oxycodone/naloxone. The HRQoL estimate based on the EQ-5D questionnaire was calculated using the weights from Dolan [7]. The indices for patient assessment of PAC-QoL and PAC-SYM scores were calculated according to Sharma and Agarwal [8].

The daily dose of oxycodone/naloxone was calculated as the average daily dose during the 4-week period if a patient had been prescribed varying doses. Costs for laxatives were calculated based on 28 days for those patients who reported the use of sodium picosulphate, polyethylene glycol or both [9]. Other pharmaceutical costs were calculated 
according to AUP prices (retail/pharmacy-level prices) from FASS (Pharmaceutical Specialties in Sweden) [10]. The unit costs for other direct costs, such as healthcare visits, telephone consultations, and inpatient days, were based on the price list for the Southern Health Care Region in 2015, which is one of the most detailed sources of information on the costs of health care resources in Sweden [11] (Table 1). All costs were calculated in Swedish krona (SEK), 2015 prices, and are presented in euros (EUR). The average exchange rates in 2015 were 1 $\mathrm{USD}=$ SEK8.435 and $1 \mathrm{EUR}=$ SEK9.356 [12].

Descriptive statistical analyses were performed to describe the patient population, including sex, age, and background pain and constipation characteristics. Descriptive analyses of variables of pharmacotherapy and resource use for the period before and after the initiation of oxycodone/naloxone were also

Table 1 Unit costs

\begin{tabular}{lc}
\hline Resource & $\begin{array}{c}\text { Cost (EUR, } \\
\text { 2015 prices) }\end{array}$ \\
\hline General practitioner & 156.26 \\
Specialist visit & 178.28 \\
Nurse visit & 82.62 \\
Telephone contact specialist physician & 72.25 \\
Telephone contact general practitioner & 52.05 \\
Telephone contact nurse & 34.63 \\
Hospitalization per day including & 505.02 \\
physician ${ }^{\mathrm{a}}$ & \\
Laxative (polyethylene glycol) & 0.38 \\
2 sachets per day & \\
Laxative (sodium picosulphate) 10 & 0.12 \\
drops per day & \\
Laxative (enemas) package $4 \times 5$ ml & 6.31 \\
\hline a Average of oncology, neurology, and orthopedic ward \\
Assumptions
\end{tabular}

performed. Continuous variables were described by the mean and standard deviation (SD) and by the median with minimum and maximum values, as appropriate. Categorical variables were reported using numbers and proportions. For the QoL variables the difference between before and after the initiation of oxycodone/naloxone was tested using a paired $t$ test. The statistical tests were two-sided, and the significance level was set as 0.05. All analyses were performed using STATA release 14 (StataCorp LP, College Station, TX).

\section{Compliance with Ethics Guidelines}

Ethical approval for the study was obtained by the Regional Ethical Board in Lund in 2013 (Dnr 2013/25 and 2013/28), and all procedures followed were in accordance with the Helsinki Declaration of 1964, as revised in 2013. Informed consent was obtained from all patients included in the study.

\section{RESULTS}

Patients were recruited from June 2013 to April 2015 from ten different clinical centers in Sweden. The majority of the participating centers were specialized pain clinics. In total, 37 patients treated with oxycodone and laxatives were included in the study, and data from both before and after the initiation of oxycodone/naloxone were available for the most central variables for 31 patients. The number of patients who responded differs between the different questionnaires. Patient characteristics are presented in Table 2.

The total number of visits to caregivers and telephone contacts was higher in the 4-week period before the initiation of oxycodone/naloxone treatment than in the 
Table 2 Patient characteristics

\begin{tabular}{lc}
\hline Variable & Patients $(\boldsymbol{n}=\mathbf{3 7})$ \\
\hline Age (years) & $61(28-88)$ \\
Sex & $11(30 \%)$ \\
$\quad$ Male & $26(70 \%)$ \\
Female & \\
Diagnosis & \\
Arthritis & $3(8 \%)$ \\
Back pain & $4(11 \%)$ \\
Malignancy & $12(32 \%)$ \\
Neuropathic pain & $1(3 \%)$ \\
Other $^{b}$ & $17(46 \%)$ \\
\hline
\end{tabular}

Values in table are presented as the mean with the minimum-maximum in parenthesis or as the number of patients with the percentage in parenthesis, as appropriate

a Primary diagnoses related to pain, several patients had more than one diagnosis

b For example, other musculoskeletal disorders, gastrointestinal disorders, and vascular disorders

4-week period immediately thereafter, although the number of nurse visits showed a slight increase during this time interval (Table 3).

Few patients had been hospitalized due to pain or constipation during the 4 weeks immediately preceding each visit. In total, two patients had spent 11 days (4 and 7 days, respectively) in hospital during the 4 weeks immediately preceding the initiation of oxycodone/naloxone treatment, and two patients had spent 8 days (4 days each) in hospital during the 4 weeks immediately following the initiation of oxycodone/naloxone.

The mean dose of oxycodone/naloxone was $39.2 \mathrm{mg} /$ day, and the daily doses of oxycodone varied between 10 and $80 \mathrm{mg}$. Since some patients had dose adjustments during the study period $(n=3)$, the mean dose was calculated as a weighted mean based on the number of days with a specific dose. The dosage of oxycodone in the participating patients ranged from low to moderate, corresponding to approximately 15-120 mg MED (morphine equivalents per day) [13]. High-dose opioid therapy has been defined as $>200 \mathrm{mg}$ MED [14], although a recent guideline considers a MED of $>90 \mathrm{mg}$ to be a high dose [2].

After the initiation of oxycodone/naloxone treatment the patients were asked to score their global impression of pain relief (global impression of oxycodone/naloxone), ranging from 1 (very poor) to 5 (very good). For the majority of the responding patients the impression of pain relief was good to very good.

Table 3 Total number of visits and telephone contacts to different caregivers due to pain and/or constipation in the 4 weeks before and 4 weeks after the initiation of oxycodone/naloxone, respectively

\begin{tabular}{|c|c|c|c|c|}
\hline \multirow[t]{2}{*}{ Type of caregiver } & \multicolumn{2}{|l|}{ Visits } & \multicolumn{2}{|l|}{ Calls } \\
\hline & Before visit $1^{a}$ & Before visit $2^{a}$ & Before visit $1^{\mathrm{a}}$ & Before visit $2^{a}$ \\
\hline Specialist physician & 10 & 6 & 12 & 0 \\
\hline General practitioner & 12 & 1 & 6 & 1 \\
\hline Nurse & 3 & 7 & 31 & 10 \\
\hline Other caregiver & 1 & 0 & 0 & 0 \\
\hline Total number & 26 & 14 & 49 & 11 \\
\hline
\end{tabular}

Total number of patients: 37. Data in table are presented as the number of visits/calls

a The observational study consisted of two visits, i.e., before (visit 1) and after (visit 2) the initiation of oxycodone/naloxone treatment, covering a total period of 8 weeks ( 4 weeks before the initiation of treatment and 4 weeks thereafter) 
Purchases of laxatives were higher before the initiation of oxycodone/naloxone treatment, with 23 patients reporting that they had bought laxatives, than after the initiation of oxycodone/naloxone treatment, with ten patients reporting such purchases.

No patients in the study had any full-time employment, and only three patients reported part-time employment. A majority of the patients reported that they were retired or have had an early retirement. Several of the patients indicated that they were on long-term sick leave.

Both HRQoL measured with the EQ-5D instrument and experience of pain intensity
EQ-VAS showed improvements after the initiation of oxycodone/naloxone, although the differences were not statistically significant. The improvements measured by the disease-specific instruments PAC-QoL, PAC-SYM, and BFI were all statistically significant (Table 4).

The direct costs per patient during each of the 4 -week periods preceding visits 1 and 2, respectively, covering resource utilization associated with treatment of OIC, with the exception of costs for analgesics and prescribed laxatives, are presented in Table 5. In general, the costs for the 4-week period after oxycodone/naloxone had been prescribed were

Table 4 Generic and disease-specific quality-of-life indicators and pain intensity before and after the initiation of oxycodone/naloxone treatment

\begin{tabular}{|c|c|c|c|c|c|c|c|c|c|}
\hline \multirow[t]{2}{*}{ Category } & \multicolumn{3}{|c|}{ Visit 1} & \multicolumn{3}{|c|}{ Visit 2} & \multirow[t]{2}{*}{$n^{\mathrm{h}}$} & \multirow[t]{2}{*}{ Difference } & \multirow[t]{2}{*}{$p$ value } \\
\hline & $n$ & Mean & SD & $\bar{n}$ & Mean & SD & & & \\
\hline EQ-5D $(0-1)^{\mathrm{a}}$ & 37 & 0.34 & 0.32 & 30 & 0.48 & 0.32 & 30 & 0.10 & NS \\
\hline EQ-5D VAS $(0-100)^{\mathrm{b}}$ & 35 & 47.43 & 18.81 & 30 & 55.77 & 20.82 & 29 & 8.55 & NS \\
\hline PAC-QoL $(0-96)^{c}$ & 37 & 44.19 & 19.12 & 31 & 28.32 & 20.82 & 31 & -16.94 & 0.000 \\
\hline \multicolumn{10}{|l|}{ Dissatisfaction index } \\
\hline PAC-QoL $(0-16)^{\mathrm{d}}$ & 37 & 4.68 & 4.08 & 31 & 7.23 & 3.97 & 31 & 2.77 & 0.009 \\
\hline \multicolumn{10}{|l|}{ Satisfaction index } \\
\hline PAC-SYM $(0-48)^{\mathrm{e}}$ & 37 & 24.54 & 8.98 & 31 & 17.42 & 10.33 & 31 & -6.71 & 0.003 \\
\hline BFI $(0-100)^{f}$ & 37 & 71.86 & 19.45 & 30 & 42.77 & 28.91 & 30 & -31.60 & 0.000 \\
\hline Pain intensity $(0-10)^{\mathrm{g}}$ & 37 & 4.84 & 2.25 & 30 & 4.07 & 2.10 & 30 & -0.43 & NS \\
\hline
\end{tabular}

$S D$ standard deviation, $N S$ not significant

${ }^{a}$ EuroQoL five dimensions (EQ-5D) instrument measures generic quality of life (QoL), with 1 being equal to 'perfect health' and 0 being equal to 'death'

b EuroQoL visual analog scale (EQ-VAS) instrument, where 100 is equal to the 'best imaginable health state' and 0 is equal to 'worst imaginable health state'

${ }^{c}$ Patient Assessment of Constipation-Quality of Life (PAC-QoL) instrument: dissatisfaction index scores range from 0 ('not at all' or 'none of the time') to 4 ('extremely' or 'all of the time'), with a maximum value of 96

d PAC-QoL instrument: satisfaction index scores range from 0 ('not at all' or 'none of the time') to 4 ('extremely' or 'all of the time'), with a maximum value of 16

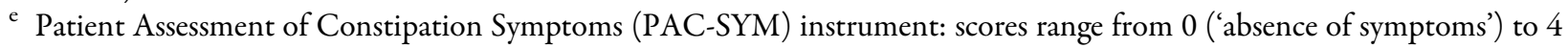
('very severe symptom'), with a maximum value of 48

${ }^{\mathrm{f}}$ Bowel Function Index (BFI) where 0 is equal to 'no problems' and 100 is equal to 'most severe problems'

g Mean pain intensity scores range from 0 ('no pain') to 10 (worst pain imaginable')

${ }^{\mathrm{h}}$ The number of patients that have values both at visit 1 and visit 2 
Table 5 Direct costs per patient during each of the 4-week periods preceding visits 1 and 2, respectively, related to pain and constipation, excluding costs of oxycodone and prescribed laxatives and costs of oxycodone/naloxone

\begin{tabular}{|c|c|c|c|}
\hline \multirow[t]{2}{*}{ Direct healthcare costs } & \multicolumn{2}{|c|}{ Direct healthcare costs (EUR, 2015 prices) } & \multirow{2}{*}{$\begin{array}{l}\text { Difference } \\
\text { (EUR) }\end{array}$} \\
\hline & $\begin{array}{l}\text { Before visit } 1 \text { (treatment with } \\
\text { oxycodone and laxatives) }(n=37)\end{array}$ & $\begin{array}{l}\text { Before visit } 2 \text { (treatment with } \\
\text { oxycodone/naloxone) }(n=37)\end{array}$ & \\
\hline Healthcare visits & 109.98 & 58.25 & 51.73 \\
\hline Hospitalization & 150.17 & 146.64 & 3.53 \\
\hline Healthcare telephone contacts & 62.31 & 18.60 & 43.72 \\
\hline $\begin{array}{l}\text { Laxative purchases } \\
\text { without prescription }\end{array}$ & 4.70 & 3.31 & 1.39 \\
\hline Total & 327.17 & 226.81 & 100.36 \\
\hline
\end{tabular}

lower than those for the initial 4-week period when patients had been treated with oxycodone and laxatives. The total difference (EUR100.36, over 28 days) between the two 4-week periods corresponds to an average cost-saving of EUR3.58/day. In comparison, the average daily cost of oxycodone/naloxone was EUR2.32, corresponding to a net cost-saving of EUR1.26 per day. In addition, the daily cost of oxycodone and laxatives in the first 4 weeks of the study period (preceding visit 1) should also be considered in order to estimate the total cost-savings.

Costs related to adverse events were not estimated because most adverse events reported were not related to treatment of pain or OIC.

Indirect costs related to productivity losses for patients who could not work due to problems with constipation were not calculated because no patient indicated that they had any full-time employment and only three patients had part-time employment. In addition, the mean age was high, such that $62 \%$ of the patients were retired. Also, several patients had malignant diseases, which probably was the reason for not working.

\section{DISCUSSION}

The results of our analysis show that in the 4 weeks immediately after the patients had started treatment with oxycodone/naloxone, resource utilization, measured as healthcare visits, telephone consultations, and purchases of non-prescribed laxatives, decreased in comparison to the 4 weeks preceding the initiation of this treatment regimen when the patients were treated with oxycodone and laxatives. The one exception to this decreasing trend in resource utilization was the slight increase in number of nurse visits between the first and second 4-week time interval. A possible explanation for this trend could be that since the number of physician visits decreased during the same period, patients' problems after oxycodone/naloxone may have been considered to be less serious and that a visit to a nurse was sufficient. The decrease in resource utilization resulted in decreased costs of EUR100.36 per patient for the 4-week period, or EUR3.58/day, compared with the average daily cost of oxycodone/naloxone of EUR2.32. At the same time there was a decrease in pain 
intensity, although the decrease was not statistically significant. There were patient-reported improvements in all QoL measures following 4 weeks of treatment with oxycodone/naloxone in comparison to the 4 weeks immediately before initiation of this treatment, but the differences were only statistically significant for the two PAC-QoL measures, PAC-SYM, and for BFI. In addition, changes in the BFI score of $\geq 12$ points are likely to be related to clinically meaningful changes in the patient's perception of their bowel habits [15]. These results are in line with those reported in other studies which have demonstrated improved QoL for patients treated with oxycodone/naloxone [16] and that oxycodone/naloxone is a cost-effective option for treating patients with severe pain and OIC $[17,18]$.

One limitation of the cost calculation in this study is that the costs of drugs prescribed during the 4-week period before the initiation of the oxycodone/naloxone treatment were not included. The reason for not including these data is that a large variety of different types of drugs and doses were reported for this time interval. Nevertheless, the cost calculation shows that treatment with oxycodone/naloxone in the analyzed patient population was cost-saving even when only including costs for hospitalization, healthcare contacts and over-the-counter purchases of laxatives. Adding the costs for prescribed drugs for the period before the initiation of oxycodone/naloxone treatment would yield an even larger cost-saving.

In spite of the high occurrence of OIC, inclusion of patients proved to be very difficult. One reason for this may be that the majority of patients referred to the participating centers had multiple medical and other problems that either excluded them from the study or, if the patients were eligible, led them to decline to participate. The main drawback of the study is the limited number of patients available for analysis. It was therefore not meaningful to perform additional statistical analyses.

\section{CONCLUSION}

The most notable conclusion which can be drawn from our results is that the oxycodone/naloxone treatment appeared to have an overall positive effect on patients. The effect consisted mainly of lessening the constipation problems of the patients, which resulted in increased HRQoL and the consumption of fewer resources. Statistically significant changes before and after initiation of oxycodone/naloxone were found in the PAC-QoL, PAC-SYM and BFI scores. The results should be interpreted with caution because of the small patient sample.

\section{ACKNOWLEDGEMENTS}

Sponsorship and article processing charges for this study were funded by Mundipharma AB, Gothenburg, Sweden.

The named authors meet the International Committee of Medical Journal Editors (ICMJE) criteria for authorship for this manuscript, take responsibility for the integrity of the work as a whole, and have given final approval to the version to be published. All authors had full access to all of the data in this study and take complete responsibility for the integrity of the data and accuracy of the data analysis.

Disclosures. Frida Hjalte, Gunnel Ragnarson Tennvall and Karl-Olof Welin declare no conflict of interest. Dagmar Westerling was 
moderator at an educational event arranged by Mundipharma in 2015.

Compliance with Ethics Guidelines. Ethical approval for the study was obtained by the Regional Ethical Board in Lund in 2013 (Dnr $2013 / 25$ and 2013/28) and all procedures followed were in accordance with the Helsinki Declaration of 1964, as revised in 2013. Informed consent was obtained from all patients being included in the study.

Open Access. This article is distributed under the terms of the Creative Commons Attribution-NonCommercial 4.0 International License (http://creativecommons.org/licenses/ by-nc/4.0/), which permits any noncommercial use, distribution, and reproduction in any medium, provided you give appropriate credit to the original author(s) and the source, provide a link to the Creative Commons license, and indicate if changes were made.

\section{REFERENCES}

1. Bruera E, Paice JA. Cancer pain management: safe and effective use of opioids. Am Soc Clin Oncol Educ Book. 2015:e593-599.

2. Dowell D, Haegerich TM, Chou R. CDC guideline for prescribing opioids for chronic pain-United States, 2016. JAMA. 2016;315(15):1624-45.

3. McNicol E. Opioid side effects and their treatment in patients with chronic cancer and noncancer pain. J Pain Palliat Care Pharmacother. 2008;22(4):270-81.

4. Hjalte F, Berggren A-C, Bergendahl H, Hjortsberg C. The direct and indirect costs of opioid-induced constipation. J Pain Symptom Manag. 2010;40(5):696-703.

5. Lowenstein $\mathrm{O}$, Leyendecker $\mathrm{P}$, Hopp $\mathrm{M}$, et al. Combined prolonged-release oxycodone and naloxone improves bowel function in patients receiving opioids for moderate-to-severe non-malignant chronic pain: a randomised controlled trial. Expert Opin Pharmacother. 2009;10(4):531-43.

6. Ahmedzai SH, Nauck F, Bar-Sela G, Bosse B, Leyendecker P, Hopp M. A randomized, double-blind, active-controlled, double-dummy, parallel-group study to determine the safety and efficacy of oxycodone/naloxone prolonged-release tablets in patients with moderate/severe, chronic cancer pain. Palliat Med. 2012;26(1):50-60.

7. Dolan P. Modeling valuations for EuroQol health states. Med Care. 1997;35(11):1095-108.

8. Sharma S, Agarwal B. Scoring systems in evaluation of constipation and obstructed defection syndrome (ODS). J Int Med Sci Acad. 2012;25(1):57-9.

9. Apoteket AB. http://www.apoteket.se. Accessed 11 Oct 2015.

10. FASS Pharmaceutical Specialties in Sweden 2015. http://www.fass.se. 2015. Accessed 11 Oct 2015.

11. Southern Regional Health Care Committee. Regional prices and payments for the Southern Healthcare Region in 2015 [Regionala priser och ersättningar för Södra Sjukvårdsregionen 2015]. http://www.skane.se/. 2015. Accessed 11 Oct 2015.

12. Sveriges riksbank (Swedish National Bank). Interests and exchange rates 2015. http://www.riksbank.se/ en/Interest-and-exchange-rates. Accessed 7 Jan 2016.

13. Brooks A, Kominek C, Pham TC, Fudin J. Exploring the use of chronic opioid therapy for chronic pain: when, how, and for whom? Med Clin N Am. 2016;100(1):81-102.

14. Chou R, Fanciullo GJ, Fine PG, et al. Clinical guidelines for the use of chronic opioid therapy in chronic noncancer pain. J Pain. 2009;10(2):113-30.

15. Rentz AM, Yu R, Muller-Lissner S, Leyendecker P. Validation of the Bowel Function Index to detect clinically meaningful changes in opioid-induced constipation. J Med Econ. 2009;12(4):371-83.

16. Morlion B, Clemens KE, Dunlop W. Quality of life and healthcare resource in patients receiving opioids for chronic pain: a review of the place of oxycodone/naloxone. Clin Drug Investig. 2015;35(1):1-11.

17. Dunlop W, Uhl R, Khan I, Taylor A, Barton G. Quality of life benefits and cost impact of prolonged release oxycodone/naloxone versus 
prolonged release oxycodone in patients with moderate-to-severe non-malignant pain and opioid-induced constipation: a UK cost-utility analysis. J Med Econ. 2012;15(3):564-75.

18. Goeree R, Goeree J. Cost-effectiveness analysis of oxycodone with naloxone versus oxycodone alone for the management of moderate-to-severe pain in patients with opioid-induced constipation in Canada. J Med Econ. 2016;19(3):277-91. 